\title{
O uso da programação linear para maximização de lucros em uma empresa de serviço de corte e dobra de aço para a construção civil
}

\author{
The use of Linear Programming for Maximizing Profits in a Steel Cutting and Bending Service
}

\author{
Company for Civil Construction
}

El uso de programación lineal para maximizar las ganancias en una empresa de servicios de corte y plegado de acero para la construcción civil

Fagner José Coutinho de Melo ORCID: https://orcid.org/0000-0002-0550-5177

Universidade de Pernambuco, Brasil

E-mail: fagnercoutinhomelo@gmail.com

Joás Tomaz de Aquino

ORCID: https://orcid.org/0000-0002-4595-5797 Instituto Federal de Pernambuco, Brasil E-mail: joastomaz@outlook.com

Camila Campos Gómez Famá

ORCID: https://orcid.org/0000-0002-7179-9644 Instituto Federal da Paraíba, Brasil E-mail: camilafama@yahoo.com.br

Calline Neves de Queiroz Claudino

ORCID: https://orcid.org/ 0000-0002-9889-7607

Universidade Estadual da Paraíba, Brasil E-mail: callineq@gmail.com

Aline Amaral Leal

ORCID: https://orcid.org/0000-0003-1673-831X

Universidade Federal de Pernambuco, Brasil E-mail: alineleal10@ hotmail.com

Taciana de Barros Jerônimo

ORCID: https://orcid.org/0000-0003-1643-3030

Universidade Federal de Pernambuco, Brasil E-mail: taciana.barros@gmail.com

\begin{abstract}
Resumo
Este artigo tem objetivo de realizar uma previsão da quantidade do serviço de Corte, Dobra e Armadura Pronta de uma empresa de corte, dobra e transporte de aço. O estudo foi realizado através da modelagem do problema na programação linear, de modo a maximizar o lucro obtido pela empresa mediante a demanda da construção civil pelo serviço e também pelas restrições identificadas na sua linha de produção, são elas: mão de obra, turno, energia, aluguel, demanda de mercado, maquinário disponível, dentre outros. Utilizou-se a ferramenta Solver, para resolução do problema. Chegou-se à conclusão que para a obtenção do lucro de 568.320,00 reais é preciso elaborar 1900 unidades corte e dobra do aço com custo de 267,66 reais, e para prestar o serviço de 100 armações o custo máximo deve ser 597,66. Desta forma, observou-se que a modelagem do problema dará maior objetividade ao retorno financeiro em detrimento aos custos operacionais.
\end{abstract}

Palavras-chave: Corte e dobra de aço; Lucro; Otimização; Programação linear; Pesquisa operacional.

\begin{abstract}
This article has aimed to make a prediction of the amount of the court service, Fold and Armor Ready for a company cutting, folding and transport of steel. The study was conducted by modeling the problem in linear programming, in order to maximize the profit made by the company upon the demand of construction for the service and also by the constraints identified in its production line, they are: labor, shift, energy, rent, market demand, machinery available, among others. We used the Solver tool to solve the problem. Reached the conclusion that to achieve the real profit is $568.320,00$ units in 1900 need to be developed cutting and bending steel with real cost of 267.66 , and to provide the 100 frames service the maximum cost should be 597.66. Thus, it was observed that the modeling of the problem will give greater objectivity to the financial return over operating costs.
\end{abstract}

Keywords: Steel bending and cutting; Profit; Optimization; Linear programming; Operational research. 


\begin{abstract}
Resumen
Este artículo tiene como objetivo hacer una previsión de la cantidad del servicio de Corte, Doblado y Armado Ready de una empresa de corte, doblado y transporte de acero. El estudio se realizó a través de la modelización del problema en programación lineal, con el fin de maximizar el beneficio obtenido por la empresa a través de la demanda de la construcción civil para el servicio y también por las restricciones identificadas en su línea de producción, son: laborales, turno, energía, alquiler, demanda del mercado, maquinaria disponible, entre otros. Se utilizó la herramienta Solver para resolver el problema. Se concluyó que para obtener una ganancia de 568.320,00 reales es necesario preparar 1900 unidades de corte y doblado de acero a un costo de 267,66 reales, y para prestar el servicio de 100 marcos el costo máximo debe ser 597,66. Así, se observó que la modelización del problema dará mayor objetividad al rendimiento financiero a expensas de los costos operativos.
\end{abstract}

Palabras clave: Doblado y corte de acero; Lucro; Mejoramiento; Programación lineal; Investigación operativa.

\title{
1. Introdução
}

A indústria da construção civil tem um papel central no processo de desenvolvimento econômico de qualquer nação (Chi Lam \& Oshodi, 2016; Jeronimo et al., 2018), isso ocorre porque essa é uma indústria que emprega no Brasil, aproximadamente, 7,6\% da população economicamente ativa (IBGE, 2016), gerando vários postos de trabalho, direta e indiretamente. Além disso, é um polo gerador de investimentos, possuindo cerca de 307 bilhões de reais de valor adicionado bruto no Brasil, em 2014 (IBGE, 2016).

Devido ao impacto desse setor na economia, ele é considerado um indicador de desenvolvimento para o país, pois seu impacto sobre o Produto Interno Bruto (PIB) e taxa de desemprego são interligados (Jeronimo \& Melo, 2015; Chi Lam \& Oshodi, 2016; Moura, 2017). Além do mais, a construção civil é capaz de gerar encadeamentos a montante e jusante em diversas cadeias produtivas, decorrente do déficit habitacional e infraestrutura ainda incipiente no Brasil.

O nível de atividade da indústria da construção civil já apresenta há algum tempo distante da normalidade devido a fase de ajuste do mercado imobiliário, do menor crescimento da renda e consumo das famílias além do encarecimento da oferta de crédito. O índice de nível de atividade na construção civil vem oscilando de acordo com a retração econômica que ocorre no Brasil. Dados da Confederação Nacional da Indústria (CNI) mostram que, apesar do recuo do setor em janeiro de 2020, o segmento mantém-se em recuperação. Na comparação com janeiro de 2019, os indicadores mostram um desempenho mais favorável da indústria da construção. Nesse período de 12 meses, o percentual médio de utilização da capacidade operacional aumentou de 55\%, em janeiro de 2019, para 60\%, em janeiro de 2020 (CNI, 2020).

Neste cenário, o consumo de aço é afetado diretamente, pois o comércio de produtos de aço destinados para a construção civil está fortemente correlacionado com o superávit ou déficit desse setor, tendo em vista que eles são um dos principais insumos para a construção civil. O sistema construtivo em aço permite liberdade no projeto de arquitetura, maior área útil, flexibilidade, compatibilidade com outros materiais, menor prazo de execução, racionalização de materiais e mão de obra, alívio de carga nas fundações, garantia de qualidade, maior organização nos canteiros de obras e precisão construtiva (Sicetel, 2014).

Contudo, na última década a produção brasileira de aço bruto ficou estagnada entre 33 e 35 milhões de toneladas/ano e a participação do Brasil na produção mundial de aço bruto caiu de $3 \%$ para 2,1\%. A baixa rentabilidade do setor siderúrgico aliada ao crescente excedente de capacidade, tanto interna quanto externamente, tem inibido e/ou postergado investimentos do setor no Brasil (Sicetel, 2014).

Desta forma, pode-se considerar que o desempenho de um mercado tem suas limitações e obstáculos influenciados pelo conjunto de instituições, normas e condições que regulam a economia de um país. Estas podem ser constituídas em forma de instituições, tais como: normas e regras informais (sanções, políticas, costumes e códigos de conduta corporativa) e formais (constituições, leis e direitos de posse e de propriedade) (North, 1990; Oliveira et al., 2020; Leal et al., 2021) Os mercados considerados eficientes institucionalizam seu ambiente mercadológico para possuir meios suficientes para que os contratos de 
prestação de serviço/ elaboração de produto sejam estabelecidos e cumpridos.

Diante do exposto surge o objetivo do trabalho que é realizar, através de um problema de programação linear, uma previsão da quantidade necessária de serviço prestado (corte e dobra e armadura pronta) para o ano de 2020 por uma empresa de corte, dobra e transporte de aço para atividades do setor da construção civil de modo a maximizar o lucro obtido pela empresa mediante restrições identificadas na sua linha de produção e também da demanda do mercado pelo serviço, dada as restrições da atual recessão econômica do país.

\section{Fundamentação Teórica}

No contexto da atual sociedade é inviável imaginar o mundo que não utilize o aço. Sua produção representa um indício do estágio de desenvolvimento econômico de um país e seu consumo desenvolve-se proporcional a construção de edifícios, fabricação de veículos, instalação de meios de comunicação e produção de equipamentos domésticos e industriais. Tais produtos são comuns no nosso dia a dia, contudo fabricá-los exigem técnicas que devem ser constantemente renovadas (Costa et al., 2007; Melo \& Aquino, 2015; Sinay et al., 2020). O mercado de aço vem passando por um momento de intensas transformações, a América Latina, por exemplo, nos últimos anos, sua produção manteve-se baixa, produzindo em torno de 60 a 70 milhões de toneladas/ano, contudo o Brasil destaca-se nesse contexto como maior produtor da região, com mais de 50\% da produção, representando quase o dobro da produção comparado com o segundo produtor que é o México (SICETEL, 2014). Apesar desse cenário, a produção de aço brasileira tem apresentado um quadro de estagnação com redução de sua produção interna e mundialmente, afetando outras áreas relacionadas a tal insumo como a construção civil, por exemplo. Apesar do setor responder por boa parcela do PIB e geração de emprego e renda diante desse quadro de baixa e instabilidade, os investimentos nessa área têm diminuído consideravelmente (Sicetel, 2014).

\subsection{Pesquisa operacional e a modelagem de problemas}

A pesquisa operacional (PO) foi utilizada pela primeira vez na Grã-Bretanha em 1938 para designar o estudo sistemático de problemas estratégicos e táticos decorrentes de operações militares durante a Segunda Guerra Mundial. Especificamente ela foi inicialmente utilizada para avaliar e reposicionar adequadamente os radares do sistema de defesa aéreo da Grã-Bretanha (Correa \& Correa, 2011). Outras aplicações da pesquisa operacional incluíam o planejamento das operações de comboio, bombardeio e guerra antissubmarino (Pereira \& da Cunha, 2019; Santos et al., 2020).

Nos tempos de hoje, a pesquisa operacional vem se consolidando e sendo aplicada em várias situações e campos do conhecimento. Um fato que contribuiu para isso foi que a quantidade de informações disponíveis cresceu exponencialmente nos últimos anos com o advento da internet, surgindo a necessidade de separar as informações de caráter relevante para se que possa criar modelos da situação que se quer analisar (Lachtermacher, 2016; Battesini et al., 2018).

Os modelos matemáticos, utilizados pela PO, constituem uma abstração simplificada da realidade, representada por um conjunto de ações e reações, a partir da qual o modelo utiliza símbolos matemáticos para representar as variáveis de decisão do sistema real (Silva \& Neto, 2020). A exatidão e captura dos aspectos essenciais dessa realidade estão associados diretamente a qualidade do modelo matemático construído (Caixeta-Filho, 2001; Battesini et al., 2018). A estruturação de um modelo, a partir da PO, está esquematizado na Figura 1. 
Figura 1: Esquematização das fases de um estudo aplicando a pesquisa operacional.

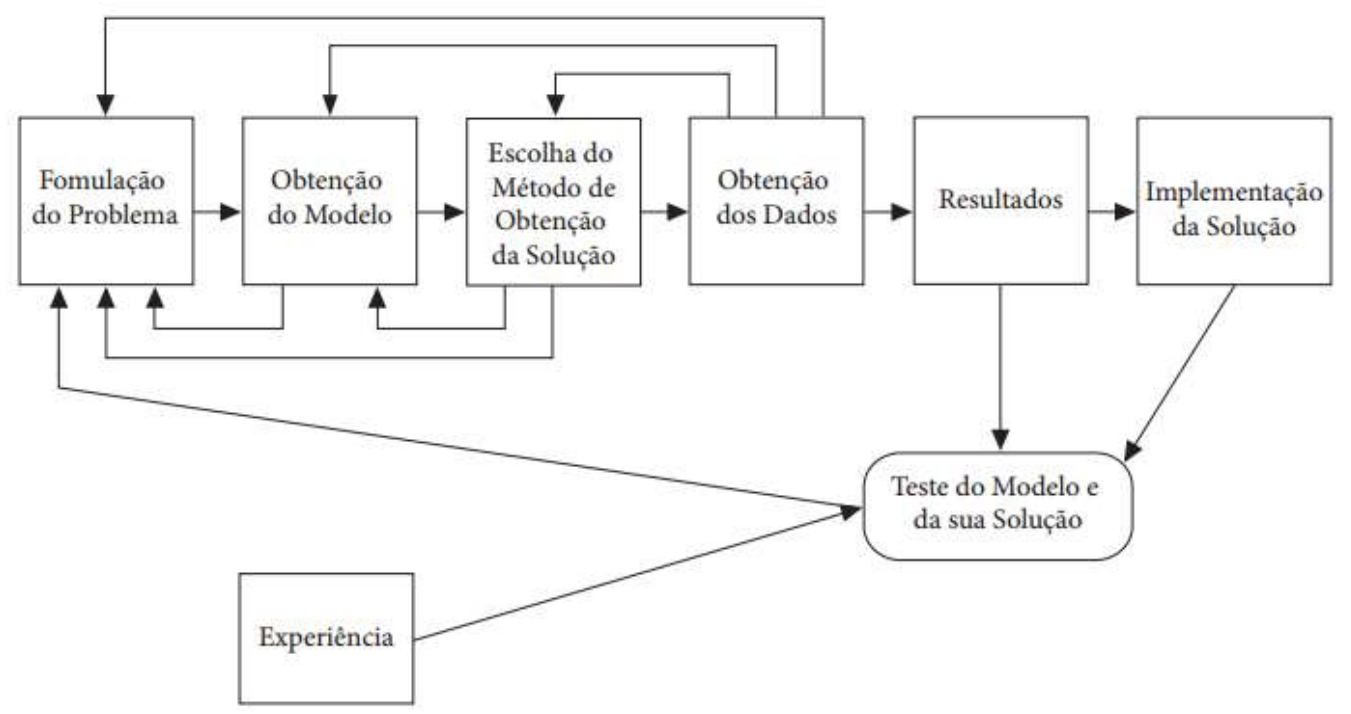

Fonte: Marins (2011).

Todo o processo inicia-se com a formulação do problema, essa etapa envolve o entendimento acerca das variáveis que compõe o problema, bem como a sua dinâmica com os possíveis externalidades. Assim, alguns questionamentos básicos podem ser evidenciados nessas etapas, tais como: quem tomará as decisões e qual o seu perfil diante da incerteza? quais são os seus objetivos? que aspectos estão sujeitos ao controle de quem decide (variáveis de decisão) e quais as limitações a que estão sujeitas essas variáveis (restrições)? quais os aspectos que estão envolvidos no processo e que fogem ao controle de quem decide? (Marins, 2011; Cenci, 2019).

Após o problema ser formulado, obtém-se o modelo a partir da abstração do problema real, nessa fase são estabelecidas as relações entre as variáveis e quais possuem maior importância. Essa abstração é feita por meio da utilização de modelos matemáticos. Vale salientar que nessa fase, de transformação da situação real em um modelo racionalizado, deve ser feita sob grande responsabilidade e cautela, pois é a partir dele que todo o processo posterior é construído e qualquer erro de modelagens prejudicará as possíveis soluções para o problema que se pretende resolver. Em seguida o decisor, levando em consideração as características do problema, escolhe o método a ser utilizado, que pode ser: programação linear, programação em redes, a Teoria dos Grafos, Teoria das Filas, métodos multicritérios, lógica fuzzy. Dada a complexidade dos problemas, muitos softwares podem ser utilizados para auxiliar o gestor (Cavalcante \& dos Santos, 2019).

Em seguida são obtidos os dados a serem utilizados no método escolhido e a interpretação dos resultados. Como os problemas possuem natureza complexa, muitas vezes, os responsáveis pela análise podem tirar conclusões errôneas e por isso, o teste do modelo e da solução obtida deve ser testada. E até mesmo na implementação da solução, a mesma deve ser testada, se necessário. O processo da utilização da pesquisa operacional para resolução de problemas não se dá de forma linear, mas sim com várias avanços e retrocessos, como mostrado na Figura 1, para uma melhor estruturação do problema e maior fidedignidade com a situação real em estudo, sempre levando em consideração a experiência do indivíduo que lida com o problema dia a dia (Botacim et al., 2019).

$\mathrm{O}$ estudo da PO, tendo em vista a sua sistemática, leva o decisor a adquirir um raciocínio organizado. Essa formalização do raciocínio facilita a análise e interpretação dos problemas reais, levando a um exame detalhado dos aspectos envolvidos para uma tomada de decisão racional (Marins, 2011). No presente estudo, será utilizada o método da programação linear (PL), que se trata de um ramo da Pesquisa operacional que descreve um sistema organizado através de um modelo matemático que contribui para o processo de análise nas tomadas de decisão, permitindo que a decisão seja muito bem avaliada 
antes de implementada (Souza et al., 2013).

\subsection{A programação linear}

A programação linear (PL) é uma das técnicas mais utilizadas em problemas de pesquisa operacional (Belfiore \& Fávero, 2012). Sua aplicação está cada vez mais difundida em ambientes organizacionais em diversas áreas e diversos setores, reflexo, sobretudo, da significativa economia gerada pelas organizações que a utilizam. A programação linear é, portanto, um método científico de tomada de decisão que descreve um sistema organizado através de um modelo matemático composto por uma função-objetivo e um conjunto de restrições técnicas, representadas por um grupo de funções lineares (Sousa et al., 2005; Hillier, 2006; Moreira, 2008; Souza et al., 2013).

A função-objetivo mede a eficiência e o desempenho do sistema. Enquanto as restrições garantem que essas soluções estejam de acordo com as limitações técnicas impostas pelo próprio sistema, que devem ser obrigatoriamente satisfeitas. Existem, ainda, outras restrições que exigem a não negatividade das variáveis de decisão, o que deverá ocorrer sempre que a técnica de abordagem for a de programação linear (Taha, 2008; Frossard, 2009). O modelo padrão de um problema de otimização da PL, sujeito a restrições pode ser apresentado, conforme as Equações (Moreira, 2008).

Função de otimização (que pode ser destinada a minimizar ou maximizar)

$$
f\left(x_{1}, x_{2}, \ldots, x_{k}\right)=a_{1} x_{1}+a_{2} x_{2}+\cdots+a_{k} x_{k}
$$

Sujeito a (restrições que será impostas a função de otimização):

$$
\begin{aligned}
& k_{11} x_{1}+c_{12} x_{2}+\cdots+c_{1 k} x_{k} \leq o u=\text { ou } \geq b_{1} \\
& k_{21} x_{1}+c_{22} x_{2}+\cdots+c_{2 k} x_{k} \leq \text { ou }=\text { ou } \geq b_{2} \\
& k_{n 1} x_{1}+c_{n 2} x_{2}+\cdots+c_{n k} x_{k} \leq o u=o u \geq b_{n} \\
& x_{i} \geq 0(i=1,2 \ldots, k)
\end{aligned}
$$

No modelo matemático mostrado anteriormente, deve-se interpretar que xi são variáveis de decisão, que devem ser todas contínuas, ou seja, devem assumir quaisquer valores em um intervalo de números reais; $\alpha$ i são os coefícientes ou parâmetros da função-objetivo; cij são os coeficientes das restrições, que podem ser expressas por equações ou inequações lineares; e o bi são os limites das restrições. Em um problema de programação linear, a função-objetivo e as restrições do modelo devem ser lineares, as variáveis de decisão devem ser contínuas e não negativas, e os parâmetros do modelo determinístico, de forma a satisfazer as seguintes hipóteses (Hillier, 2006; Belfiore \& Fávero, 2012):

- Proporcionalidade: requer para cada variável de decisão considerada a sua contribuição em relação à função-objetivo e às restrições do modelo seja diretamente proporcional ao valor da variável de decisão;

- Aditividade: o valor total da função-objetivo ou de cada função de restrição de um modelo de programação linear é expresso pela soma das contribuições individuais de cada variável de decisão;

- Divisibilidade: cada uma das variáveis de decisão pode assumir quaisquer valores não negativos dentro de um intervalo, incluindo valores fracionários, desde que satisfaçam as restrições do modelo;

- Certeza: conforme essa hipótese os coeficientes da função-objetivo, os coeficientes das restrições e os termos independentes de um modelo de programação linear são determinísticos.

Portanto, busca-se, dentro do conjunto das soluções viáveis, a solução ótima do modelo (Belfiore \& Fávero, 2012). 
Com esse objetivo, diversos algoritmos foram criados, destacando-se o método Simplex como o mais conhecido e utilizado (Caixeta-Filho, 2001; Belfiore \& Fávero, 2012).

\subsection{Método Simplex}

O Simplex é um algoritmo mais utilizado para a solução de problemas de programação linear (Moreira, 2008; Belfiore \& Fávero, 2012). A origem do método Simplex para resolução de problemas de programação linear data 1947, a partir da disseminação da pesquisa operacional, utilizada inicialmente para fins bélicos, nos Estados Unidos depois da Segunda Guerra Mundial, para solucionar o problema de alocação de recursos limitados, de modo a otimizar objetivos (Loesch \& Hein, 2009; Belfiore \& Fávero, 2012; Santos et al., 2019).

O algoritmo Simplex é um método iterativo que parte de uma solução básica factível inicial e busca, a cada iteração, uma nova solução básica factível adjacente, com melhor valor na função objetivo, até que o valor ótimo seja atingido Loesch \& Hein, 2009; Belfiore \& Fávero, 2012). A partir de uma solução básica atual, uma variável não básica entra na base no lugar de outra variável básica que passa a ser não básica, gerando uma solução básica adjacente. Caso a solução básica adjacente atende as restrições de não negatividade ela é denominada de solução básica factível adjacente (SBF) (Belfiore \& Fávero, 2012; Antunes et al., 2019). O algoritmo Simplex pode ser simplificado conforme a Figura 2 (Lachtermacher, 2009).

Inicialmente o problema deve estar na sua forma padrão. Em seguida deve ser encontrada uma solução básica factível (SBF) inicial para o problema de PL, que pode ser obtida atribuindo valores iguais a zero às variáveis de decisão. Efetua-se, então, o teste de otimalidade, ou seja, uma solução básica factível é ótima caso não existam soluções adjacentes melhores (Hillier, 2006).

Figura 2: Descrição do algoritmo Simplex.

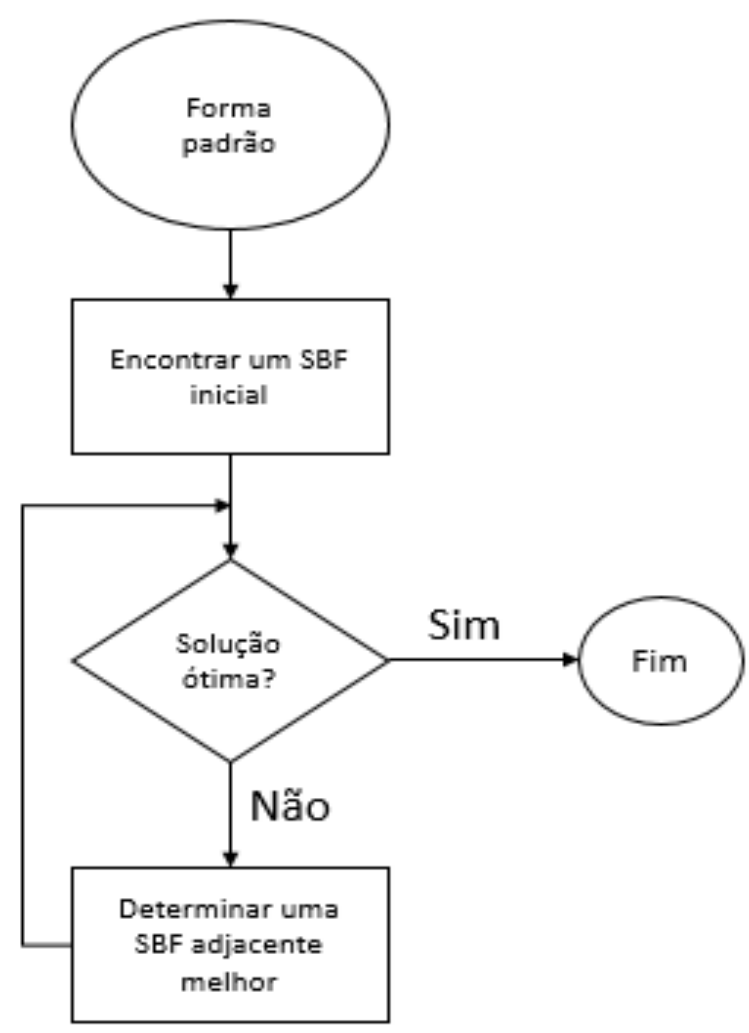

Fonte: Lachtermarcher (2009). 


\section{Metodologia}

Esta pesquisa tem por finalidade aplicar as técnicas de modelagem e programação linear sobre o problema de maximização de lucro de uma empresa de serviço de Corte e dobra de aço e armadura pronta, situada na região metropolitana do Recife, Pernambuco. Esta pesquisa pode ser classificada como estudo de caso uma vez que busca investigar em profundidade um ou mais objetos de análise com finalidade de adquirir amplo conhecimento destes (Yin, 2015). Para efeito de estudo foram obtidos os dados a respeito da composição de cada um dos tipos de serviço, o corte e dobra de aço e a armadura pronta, assim como o preço do serviço, a capacidade produtiva, mão de obra disponível, turno de trabalho e entrega de matéria prima. A partir destas informações, determinou-se um uma função objetivo a fim de maximizar os lucros da organização estudada.

\subsection{Identificação da unidade produtiva}

A empresa estudada é uma unidade de serviço de corte e dobra de aço e de armaduras prontas fabricadas em centrais de serviços especializados, no qual busca diminuir o quantitativo de mão de obra e equipamentos nos canteiros de obra.

Nas centrais de serviço, para a atividade de corte e dobra, são fabricadas peças de elementos estruturais, que serão utilizadas em vigas, lajes, pilares e etc., em equipamentos tecnologicamente avançados garantindo a precisão em todos os formatos de peças cortadas e dobradas. A atividade da armadura pronta supera o serviço anteriormente mencionado, nesta atividade a empresa entrega o elemento estrutural já finalizado e armado para a execução da etapa seguinte em obra, todos os serviços citados acima são entregues de acordo com os projetos enviados pela própria obra.

Os serviços em aço estão apontando uma nova tendência mundial (Grandes Construções, 2019). A industrialização dos serviços na da construção civil e a racionalização dos seus processos nos canteiros de obra estão a demanda por trabalhadores na construção e ajustando cronogramas de obra em tempos escassos de mão de obra e tempo.

No serviço de corte e dobra as peças dos elementos estruturais são entregues conforme a necessidade, especificação e solicitação do cliente, sendo estes identificados com etiquetas indicando o nome do elemento estrutural ao qual pertence, posição, desenho do formato e dimensão, entre outros, ou seja, possuindo todas as informações necessárias à montagem da encomenda. O serviço de corte e dobra de aço supre as necessidades das obras trazendo claros benefícios como a redução de perda de aço, assistência técnica especializada, gerenciamento na execução de estruturas, diminuição de custos, racionalização do canteiro de obra, aumento de produtividade e construção otimizada.

Para a atividade da armadura pronta, após a realização do corte e dobra do aço, de acordo com todas as especificações dos clientes, todo aço é montado e soldado previamente, no processo de soldagem é utilizado a solda MIG/MAG seguindo os critérios do controle da qualidade, garantindo rigidez a armadura. As armaduras são entregues conforme a solicitação do cliente direto na obra de acordo com o seu cronograma. Desta forma, a o serviço de armadura pronta busca proporcionar a redução de fases na obra, a otimização nos canteiros e diminuição dos riscos de acidentes, além de facilitar o planejamento do cronograma da obra e o seu fluxo de caixa.

\subsection{Função objetivo do problema de otimização}

A função objetivo do problema pode ser caracterizada como uma função linear. A equação mostra uma relação entre a quantidade produzida dos serviços de corte e dobra e de armação das estruturas de aço com seus respectivos lucros. Neste sentido, a função busca maximizar o lucro obtido pela prestação dos dois serviços citados.

As Tabelas 1 e 2 mostram, respectivamente, custo e custo por tonelada mensais para os serviços de armação e corte e dobra, além do custo fixo para a execução das atividades. 
Tabela 1: Custo dos serviços de armação, corte e dobra e custo fixo para a execução das atividades.

\begin{tabular}{cc}
\hline & Custos \\
\hline Armação & 6 operários x 3 turnos x R $\$ 3000,00 /$ operário $=\mathbf{R} \$ \mathbf{5 . 4 0 0 0 , 0 0}$ \\
Corte e dobra & 10 operários x 3 turnos x R $\$ 2364,00 /$ operário $=\mathbf{R} \$ \mathbf{7 . 0 9 2 0 , 0}$ \\
& $\mathrm{R} \$ 1000,00$ (aluguel) $+\mathrm{R} \$ 60000,00$ (mão-de-obra indireta) + \\
Custo fixo & $\mathrm{R} \$ 200000,00$ (energia) $=\mathbf{R} \mathbf{2 7 0 . 0 0 0 , 0 0}$ \\
\hline
\end{tabular}

Fonte: Autores (2021).

Para o cálculo da Tabela 2, utilizaram-se as capacidades da empresa de produção do serviço de armação de 100 toneladas por mês e de execução do serviço de corte e dobra de 2100 toneladas por mês, que seriam 2000 ton/mês acrescidos de 100 ton/mês realizados na armação.

Tabela 2: Custo por tonelada dos serviços de armação, corte e dobra e custo fixo para a execução das atividades.

\begin{tabular}{|c|c|}
\hline \multicolumn{2}{|r|}{ Custos/Tonelada } \\
\hline Armação & $(\mathrm{R} \$ 3000,00 \times 6$ operários $\times 3$ turnos $) / 100$ toneladas $=\mathbf{R} \$ \mathbf{5 4 0 , 0 0 / t o n e l a d a}$ \\
\hline Corte e dobra & $(\mathrm{R} \$ 2364,00 \times 10$ operários $\times 3$ turnos $) / 2100$ toneladas $=\mathbf{R} \$ \mathbf{3 3}, \mathbf{7 7} /$ tonelad \\
\hline Custo fixo & $\mathrm{R} \$ 270000,00 / 2100$ toneladas $=\mathbf{R} \$ \mathbf{1 2 8 , 5 7 / \text { tonelada }}$ \\
\hline
\end{tabular}

Fonte: Autores (2021).

Considerando x 1 como a variável correspondente ao serviço de corte e dobra e x2 como o serviço de armação, tem-se na Equação 6 modelo de função objetivo do problema em questão.

$$
\operatorname{Max} L=(430,00-33,77-128,57) x_{1}+(1300,00-540,00-33,77-128,57) x_{2}
$$

Os valores obtidos para o luro de cada serviço são apresentados detalhadamente na Tabela 3.

Tabela 3: Preços para cálculo do lucro dos serviços prestados na empresa.

\begin{tabular}{cccc}
\hline & Preço da prestação do serviço & Custo/ton & Custo fixo \\
\hline Corte e dobra & 430,00 & 33,77 & 128,57 \\
Armação & $1.300,00$ & 540,00 & \\
\hline
\end{tabular}

Fonte: Autores (2021).

\subsection{Restrições da otimização}

A maximização do lucro está sujeita a restrições de capacidade produtiva mensal do serviço de corte e dobra e da armação e das horas mensais disponíveis para os respectivos serviços. A capacidade mensal de produção do serviço de corte e dobra é de 2000 toneladas, sendo assim a primeira restrição pode ser descrita como a Inequação a seguir.

$$
x_{1} \leq 2000
$$

O serviço de armação também se encontra limitado pela sua capacidade de produção de 100 toneladas por mês (Inequação abaixo).

$$
x_{2} \leq 100
$$

A terceira restrição se refere ao material disponível para ser trabalhado e pode ser representada pela seguinte 
Inequação.

$$
x_{1}+x_{2} \leq 2000
$$

As duas últimas restrições referem-se às quantidades de homens-horas trabalhadas. A Tabela 4 mostra o cálculo da produção em toneladas por hora e por funcionário.

Tabela 4: Produtividade por hora e por funcionário.

\begin{tabular}{cc}
\hline \multicolumn{2}{c}{ Cálculo da produtividade (ton/(h x func) } \\
\hline Corte e dobra & 2100 toneladas / $(220$ horas x 10 funcionários $)=0,96$ \\
Armação & 100 toneladas / $(220$ horas x 6 funcionários $)=0,076$ \\
\hline
\end{tabular}

Fonte: Autores (2021).

Na Tabela 5 verifica-se o cálculo da limitação de horas disponíveis para trabalho na empresa.

Tabela 5: Horas disponíveis para trabalho.

\begin{tabular}{cc}
\hline & Quantidade de horas disponíveis \\
\hline Corte e dobra & $6 \mathrm{~h} /$ turno x 3 turnos/dia x 6 dias/sem x 4 sem/mês x 10 func $=4320$ \\
Armação & $6 \mathrm{~h} /$ turno x 3 turnos/dia x 6 dias/sem x 4 sem/mês x 6 func $=2592$ \\
\hline
\end{tabular}

Fonte: Autores (2021).

A restrição referente ao serviço de corte e dobra é representada pela Inequação a seguir. Vale salientar que para realizar a armadura, anteriormente o aço deve passar pelo processo de corte e dobra.

$0,96 x_{1}+0,96 x_{2} \leq 4320$

Por fim, a restrição da quantidade de homens-hora trabalhadas para o serviço de armadura é representada pela Inequação abaixo.

$0,076 x_{2} \leq 2592$

As variáveis $\mathrm{x} 1$ e $\mathrm{x} 2$ ainda devem ser maiores ou iguais a zero.

\subsection{Uso da ferramenta Solver do Excel}

Este modelo foi solucionado utilizando-se a ferramenta Solver, do Microsoft Excel, para resolução de problemas de otimização. No estudo em questão, tem-se um problema de maximização e as restrições foram apresentadas anteriormente. À ferramenta, configurou-se a função objetivo na célula na qual se quer o resultado, e adicionaram-se as restrições referentes às células variáveis. Além disto, definiu-se no solver, o método de resolução simplex para programação linear. A Tabela 6 apresenta a solução ótima encontrada para o problema (célula destino). 
Tabela 6: Resolução do problema através da ferramenta Solver do Excel.

\begin{tabular}{|c|c|c|c|c|}
\hline Variáveis de decisão & Corte e Dobra & Armadura & & \\
\hline Cel variáveis & 1900 & 100 & & \\
\hline Função Objetivo & Corte e Dobra & Armadura & & \\
\hline Coeficiente & 267,66 & \multirow[t]{2}{*}{597,66} & & \\
\hline Cel destino & 568320 & & & \\
\hline Restrições & Corte e Dobra & Armadura & LHS & $\mathrm{RHS}=\mathrm{b}$ \\
\hline Capacidade Corte e Dobra & 1 & 0 & 1900 & 2000 \\
\hline Capacidade de Armadura & 0 & 1 & 100 & 100 \\
\hline Capacidade Conjunta & 1 & 1 & 2000 & 2000 \\
\hline H.H Disponível de Corte e Dobra & 0,96 & 0,96 & 1920 & 4320 \\
\hline H.H Disponível de Armadura & 0 & 0,076 & 7,6 & 2592 \\
\hline
\end{tabular}

Fonte: Autores (2021).

\section{Resultados e Discussão}

O preço-sombra para um determinado recurso mede o valor marginal deste recurso em relação ao lucro total. Em outras palavras, a quantidade que o lucro total (L) poderia ser melhorado, caso a quantidade do recurso puder e for aumentado a uma quantidade igual a uma unidade.

Neste caso, se fosse disponibilizada maior quantidade de recurso, este deveria ser alocado inicialmente para aumentar a capacidade de executar o serviço de armadura, pois a cada unidade aumentada deste recurso, teríamos um acréscimo de $\mathrm{R} \$$ 330 em nosso lucro. Vale salientar que essa restrição só pode ser ampliada em até 1900 unidades.

O material disponível para ser trabalhado nos serviços de corte e dobra e de armadura referente à terceira restrição também poderia ser aumentado em até 100 unidades, elevando o lucro em $\mathrm{R} \$ 267,66$ para cada unidade aumentada.

No que diz respeito ao acréscimo e decréscimo permissível, estes mostram até onde podemos mudar um coeficiente na função objetivo sem causar mudança nos valores ótimos das variáveis de decisão, ou mudar o limite da restrição de uma equação sem fazer com que quaisquer dos valores ótimos dos preços sombras ou custo reduzido mudem. A análise de sensibilidade obtida para o problema encontra-se na Tabela 7. 
Tabela 7: Análise de sensibilidade obtida através da ferramenta Solver - Excel.

\begin{tabular}{|c|c|c|c|c|c|c|}
\hline \multicolumn{7}{|c|}{ Células variáveis } \\
\hline Célula & Nome & Final valor & $\begin{array}{c}\text { Reduzido } \\
\text { custo }\end{array}$ & $\begin{array}{c}\text { Objetivo } \\
\text { Coeficiente }\end{array}$ & $\begin{array}{l}\text { Permitido } \\
\text { Aumentar }\end{array}$ & $\begin{array}{c}\text { Permitido } \\
\text { Reduzir }\end{array}$ \\
\hline$\$ B \$ 4$ & Cel. Variáveis Corte e Dobra & 1900 & 0 & 267,66 & 330 & 267,66 \\
\hline$\$ C \$ 4$ & Cel. Variáveis Armadura & 100 & 0 & 597,66 & $1 \mathrm{E}+30$ & 330 \\
\hline \multicolumn{7}{|c|}{ Restrições } \\
\hline Célula & Nome & Final valor & $\begin{array}{c}\text { Reduzido } \\
\text { custo }\end{array}$ & $\begin{array}{c}\text { Objetivo } \\
\text { Coeficiente }\end{array}$ & $\begin{array}{l}\text { Permitido } \\
\text { Aumentar }\end{array}$ & $\begin{array}{c}\text { Permitido } \\
\text { Reduzir }\end{array}$ \\
\hline \$D\$11 & Capacidade Corte e Dobra LHS & 1900 & 0 & 2000 & $1 \mathrm{E}+30$ & 100 \\
\hline$\$ D \$ 12$ & Capacidade de Armadura LHS & 100 & 330 & 100 & 1900 & 100 \\
\hline$\$ D \$ 13$ & Capacidade Conjunta LHS & 2000 & 267,66 & 2000 & 100 & 1900 \\
\hline \$D\$14 & H.H Disponível de Corte e Dobra LHS & 1920 & 0 & 4320 & $1 \mathrm{E}+30$ & 2400 \\
\hline \$D\$15 & H.H Disponível de Armadura LHS & 7,6 & 0 & 2592 & $1 \mathrm{E}+30$ & 2584,4 \\
\hline
\end{tabular}

Fonte: Autores (2021).

\section{Conclusão}

O objetivo do trabalho foi elaborar a previsão da quantidade necessária de dois tipos de serviço prestados por uma empresa de corte, dobra, armação e transporte de aço. Cujo sua principal demanda está localizada no setor da construção civil, como este setor demonstra desaceleração é oportuno identificar a quantidade de itens para a condução com qualidade do serviço. Ele foi desenvolvido através de um problema de programação linear, de modo a maximizar o lucro da empresa mediante as restrições identificadas na sua linha de produção e também da demanda do mercado pelo serviço.

Um dos problemas recorrentes é realizar a programação de produção do corte, dobra e armação do aço, ou seja, estabelecer qual a quantidade a produzir, para se obter o maior lucro possível através das limitações do sistema em estudo, como por exemplo: mão de obra, turno, energia, aluguel, demanda de mercado, maquinário disponível, dentre outros.

Desta forma, o presente estudo procurou encontrar a melhor distribuição possível, no Solver da Planilha Excel, dos recursos da empresa estudo de caso, sujeitos às limitações, de forma a atingir o valor ótimo ao objetivo estabelecido. Através da problematização do estudo chegou-se a conclusão que a para fazer 1900 unidades corte e dobra do aço o melhor custo é de 267,66 reais, e para prestar o serviço de elaboração de 100 armações o custo máximo deve ser 597,66. No sistema, estes dados foram usados para a obtenção do lucro bruto de 568.320,00 reais.

Diante deste cenário, pode ser observado que a modelagem do problema na Programação Linear é complexa, porém o seu cálculo para achar a solução ótima é bastante simples e tem maior objetividade de achar adequadamente o retorno maximizado para cada requisito do cliente da empresa estudo de caso. Assim, também é possível analisar todo o ciclo de cada empreendimento de corte, dobra e armação do aço para a construção civil.

O trabalho realizado é justificado pela maximização do lucro para a empresa que deve ser monitorado frente a mudanças do mercado consumidor, como a recessão na construção, onde se torna importante verificar o quanto seria permitido reduzir nas capacidades de produção, mantendo os valores ótimos de lucro da empresa.

Como proposta de trabalho futuro, sugere-se desenvolver um modelo de programação linear, acrescentando novas variáveis do tipo rota de transporte e custo desta rota afim de trazer um valor de lucro mais real para a organização. Outra proposta de trabalhos futuros é buscar otimizar os processos por meio da engenharia de métodos utilizando modelos de otimização de trabalho.

Desta maneira, o trabalho buscou contribuir para o conhecimento da ciência por meio de um problema estruturado a 
partir da programação linear na área da construção civil nesse cenário de recessão econômica do país. Assim, a aplicação da programação linear pode auxiliar os gestores do setor da construção, neste caso especificamente do segmento de serviços de corte e dobra do aço, no processo de tomada de decisão, sobre como gerir os custos de seus produtos, quais materiais se devem comprar, como incrementar a mão de obra para diminuir o pagamento de horas extras, entre outros. No entanto, é necessário o acompanhamento constante do desenvolvimento do modelo, para que possa ser adaptado e alinhado sempre que ocorram alterações no mercado.

\section{Referências}

Antunes, B., Gomes, N. S., Guerra, T. E., de Oliveira, R. T., \& de Boer Garbin, F. G. (2019). Maximização da receita de uma microempresa de chocolates artesanais por meio da pesquisa operacional. Anais do Salão Internacional de Ensino, Pesquisa e Extensão, 11(2).

Battesini, M., Coelho, H. D. S., \& Seta, M. H. D. (2018). Uso de programação linear para otimizar o acesso geográfico em redes temáticas de atenção à saúde. Cadernos de Saúde Pública, 34, e00055017.

Belfiore, P., Fávero, L. P. (2012). Pesquisa Operacional para cursos de administração, contabilidade e economia, Elsevier

Botacim, R. S., Fontana, V. S., Xavier, B. M., \& de Souza, M. (2019). Pesquisa Operacional: a comparação de dois métodos para resolução de um problema de programação linear. Exatas \& Engenharias, 9(24).

Caixeta-Filho, J.V. (2001). Pesquisa Operacional, Atlas.

Cavalcante, D. M., \& dos Santos, E. S. (2019). Utilização da programação linear para maximização dos lucros da produção de pães em uma empresa de panificação. Brazilian Journal of Development, 5(12), 28501-28511.

Cenci, V. R. (2019). Otimização de receita de uma escola de idiomas através do uso da programação linear. Brazilian Journal of Development, 5(12), 3077330783.

Chi Lam, K., \& Oshodi, O. S. (2016). Forecasting construction output: A comparison of artificial neural network and Box-Jenkins model. Engineering, Construction and Architectural Management, 23(3),302-322.

CNI (Confederação Nacional da Indústria). (2020). Sondagem Indústria da Construção. https://www.portaldaindustria.com.br/estatisticas/sondagem-industriada-construcao/.

Costa, V. L.; Escorsim, S.; Costa D. L. (2007). Processo produtivo e produção de aço: a inserção do Grupo Gerdau S.A. no cenário mundial. In: Congresso Internacional de Administração, 20, 2007, Anais ... Ponta Grossa, PR, Anais.

Correa, H. L.; Correa, C. A. (2011). Administração de produção e operações manufatura e serviços: uma abordagem estratégica. 2. ed. São Paulo: Atlas.

Frossard, M. C. (2009). Qualidade de Vida no Serviço Público - Um Programa para a Agência Nacional do Petróleo, Gás Natural e Biocombustíveis - ANP. Dissertação de Mestrado em Administração Pública - Fundação Getúlio Vargas. Rio de Janeiro.

Grandes Construções. (2019). http://www.grandesconstrucoes.com.br/br/index.php?option=com_conteudo\&task=viewNoticia\&id=11890.

Hillier, F. S. (2006). Introdução à Pesquisa Operacional, McGraw-Hill.

IBGE (Instituto Brasileiro De Geografia E Estatística). (2016) Estatística, I. IBGE | Séries Estatísticas \& Séries Históricas.

Jeronimo, T. B., Melo, F. J. C., Aquino, J. T., Albuquerque, A. P. G., \& Medeiros, D. D. (2018). Knowledge management alignment to the community of practice in a company of cutting and bending steel. Brazilian Journal of Operations \& Production Management, 15, 1-11.

Jeronimo, T. B. \& Melo, F. J. C. (2015). Avaliação da restrição da empresa através da teoria das restrições e da programação linear: um estudo de caso de maximização de lucro para empresa de serviço de corte e dobra de aço. Revista eletrônica eng tech science, 3, 01-21.

Lachtermacher, G. (2009). Pesquisa Operacional na Tomada de Decisões - Modelagem em Excel, Campus.

Lachtermacher, G. (2016). Pesquisa Operacional na Tomada de Decisões, Campus.

Leal, M. S. F., Melo, F. J. C., Sobral, E. F. M., \& Carneiro, J. S. (2021). Planejamento e âncora de carreira: um estudo com discentes do curso de Administração da Universidade de Pernambuco - campus Salgueiro. Research, society and development, 10, e17410212350.

Loesch, C., Hein, N. (2009). Pesquisa Operacional - Fundamentos e Modelos. Saraiva.

Marins, F. A. S. (2011). Introdução à Pesquisa Operacional. São Paulo: Cultura Acadêmica: Universidade Estadual Paulista, Pró-Reitoria de Graduação.

Melo, F. J. C., \& Aquino, J. T. (2015). Os 5S como diferencial competitivo para o sistema de gestão da qualidade: estudo de caso de uma empresa de aços longos. Gestão.Org, 13, 176-186.

Moreira, D. A. (2008). Administração de produção e operações. (2a ed.), Cengage Learning. 
Research, Society and Development, v. 10, n. 3, e32110313402, 2021

(CC BY 4.0) | ISSN 2525-3409 | DOI: http://dx.doi.org/10.33448/rsd-v10i3.13402

Moura, G. C. (2017). Previsão do custo unitário básico por meio de modelos de previsão da classe geral ARIMA. 57 f. Trabalho de Conclusão de Curso (Graduação em Engenharia Civil) - Universidade Regional do Noroeste do Estado do Rio Grande do Sul, Santa Rosa.

North, D., (1990). Institutions, Institutional Change and Economic Performance, Cambridge University Press.

Oliveira, R. P., Araujo Junior, A. H., Barros, J. G. M., Sampaio, N. A. S., \& Silva, J. W. J. (2020). Update study of the ISO 9001 standard in a capital goods company. Research, Society and Development, 9(8), e51985354.

Pereira, M. R., \& da Cunha, R. F. (2019). Aplicação do simplex para a otimização de lucratividade de um posto de combustível. Brazilian Journal of Development, 5(7), 8833-8844.

Santos, M., Silva, K. D. O. D., dos Reis, M. F., \& Martins, E. R. (2019). Apoio à decisão de um microempreendedor individual por meio do método Simplex. Revista de Trabalhos Acadêmicos Lusófona, 2(2), 305.

Santos, R. P. M., Camargo, P. R., Araujo Junior, A. H., Barros, J. G. M., Sampaio, N. A. S., Silva, J. W. (2020). Use of statistics as a decision-making tool in a technical feasibility study of the post stirring method in a steel plant. Research, Society and Development, 9(7), e498974163.

SICETEL (Sindicato Nacional Da Indústria, Trefilação e Laminação de Metais Ferrosos). (2014). Relatório: Análise do Mercado do Aço.

Silva, F. N. \& Neto, J. V. F. (2020). Tuning heuristics and convergence analysis of reinforcement learning algorithm for online data-based optimal control design. Research, Society and Development, 9(2), e188922128.

Sinay, M. C. F., Sinay, L., Braga, I. L., Martins, A. C., \& Duarte, A. L. F. (2020). Public administration and inspection of concessionaires: a case study.Research, Society and Development, 9(8), e459985837.

Souza, C. C., Reis Neto, J. F., \& Wolf, R. (2013). Utilização da Programação Linear associada à Teoria das Restrições como ferramenta no processo decisório em atividades de gestão agroindustrial. FACEF Pesquisa: Desenvolvimento e Gestão, 16, 269-280.

Sousa, R. S., Silva, C. T. L. \& Arenales, M. N. (2005). Métodos do Tipo Dual Simplex para Problemas de Otimização Linear Canalizados. Pesquisa Operacional, 25, 349-382.

Taha, H. A. (2008). Pesquisa Operacional, Pearson.

Yin, R.K. (2015). Estudo de Caso: planejamento e métodos. (5a ed.), Bookman. 